\title{
Musculoskeletal manifestations in hyperlipidaemia: a controlled study
}

\author{
P Klemp, Anne M Halland, F L Majoos, Krisela Steyn
}

\begin{abstract}
Eighty eight patients with hyperlipidaemia (81 white patients from South Africa and seven patients of mixed race from the West Cape area) were studied. Forty eight had adult familial hypercholesterolaemia, 16 had juvenile familial hypercholesterolaemia, and 24 had mixed hyperlipidaemia (increased cholesterol and triglycerides). They were interviewed and examined and their musculoskeletal manifestations compared with 88 controls with normal lipid profiles, and matched for age, sex, and race for each group of patients. The following manifestations were significantly increased in the patients: $(a)$ tendon xanthomas particularly of the tendo Achillis in patients with adult familial hypercholesterolaemia and mixed hyperlipidaemia; (b) tendo Achillis tendinitis in patients with adult familial hypercholesterolaemia and mixed hyperlipidaemia; and $(c)$ oligoarthritis in patients with mixed hyperlipidaemia but not in those with adult familial hypercholesterolaemia. Migratory polyarthritis and transient tendo Achillis pain were rare. Thirty eight per cent of patients with juvenile familial hypercholesterolaemia had musculoskeletal system manifestations none of which was significantly increased compared with controls. There was a significant association between tendon xanthomas and tendo Achillis tendinitis. There was a significant difference in pretreatment cholesterol levels in the patients with adult familial hypercholesterolaemia and musculoskeletal system manifestations compared with those without and in all three groups combined. The study confirms an association between hyperlipidaemia and tendon xanthomas, tendo Achillis tendinitis, and to a lesser extent oligoarthritis but not migratory polyarthritis or transient tendo Achillis pain as reported in other studies. It also shows that musculoskeletal system manifestations antedated the diagnosis of hyperlipidaemia in 24/39 (62\%) patients and that the manifestations improved or resolved completely in $19 / 30(63 \%)$ patients after receiving lipid lowering treatment. It is therefore important to recognise the association between musculoskeletal system manifestations and hyperlipidaemia for diagnostic and therapeutic reasons.
\end{abstract}

(Ann Rheum Dis 1993; 52: 44-48)

A number of studies suggest that there is an association between hyperlipidaemia and tendon xanthomas, tendo Achillis tendinitis, transient tendo Achillis pain, migratory poly- arthritis, and oligoarthritis. ${ }^{1-\gamma}$ The reported prevalence of these manifestations varies widely from study to study because of differences in classification of the hyperlipidaemias, patient selection, and study design. Interpretation of the findings, none of which included control patients, is difficult especially where prevalence figures are low. Migratory polyarthritis appears to be particularly associated with homozygous familial hypercholesterolaemia. ${ }^{1}$ There are, however, other uncontrolled studies which show no specific associations between hyperlipidaemia and rheumatic disorders. ${ }^{9}$ 10

In the one controlled study the only difference between patients with hypercholesterolaemia and controls was that pain, particularly of the ankles and feet, was significantly more severe in the patients. ${ }^{11}$ There was no difference in the duration of morning stiffness, analgesic use, or effect on lifestyle. This study cannot be compared with others, however, as specific entities such as xanthomas and migratory polyarthritis were not reported.

We documented the musculoskeletal system manifestations of 88 patients with hyperlipidaemia and compared them with 88 age and sex matched controls. The patients were subdivided into groups with different lipid profiles. The onset of the manifestations in relation to the diagnosis of hyperlipidaemia and the effect of lipid lowering treatment on the manifestations were also investigated.

Patients and methods

Eighty eight consecutive patients with hyperlipidaemia attending the lipid clinic at Tygerberg Hospital were studied. They were interviewed with a detailed questionnaire and examined, and the following information was recorded: age, sex, family history of hyperlipidaemia or ischaemic heart disease, musculoskeletal system manifestations past or present, cholesterol and triglyceride levels in patients with and without musculoskeletal system manifestations, the effect of lipid lowering treatment on musculoskeletal system manifestations, and whether the onset of musculoskeletal system disorders antedated or followed the diagnosis of hyperlipidaemia. Particular attention was paid to a history of migratory polyarthritis, oligoarthritis, tendo Achillis tendinitis, and transient tendo Achillis pain.

CLASSIFICATION OF HYPERLIPIDAEMIA

Patients were classified as follows. (a) Hypercholesterolaemia without hypertriglyceridaemia 
in patients at least 16 years old if (i) pretreatment total cholesterol was greater than or equal to 6.5 $\mathrm{mmol} / \mathrm{l}$, (ii) normal triglyceride levels $(\leqslant 2 \cdot 0$ $\mathrm{mmol} / \mathrm{l}$ ), (iii) strong family history of ischaemic heart disease or familial hypercholesterolaemia. This group of patients was considered to have type IIa adult familial hypercholesterolaemia. (b) Hypercholesterolaemia without hypertriglyceridaemia in patients younger than 16 years if (i) pretreatment total cholesterol was greater than or equal to $6.5 \mathrm{mmol} / \mathrm{l}$, and (ii) and (iii) as for adult familial hypercholesterolaemia. These patients are referred to as having juvenile familial hypercholesterolaemia. (c) Mixed hyperlipidaemia if (i) total cholesterol was greater than or equal to $6.5 \mathrm{mmol} / \mathrm{l}$, and (ii) triglyceride levels were greater than or equal to $2.0 \mathrm{mmol} / \mathrm{l}$. A family history of hyperlipidaemia or ischaemic heart disease was not considered in this group.

Of the 88 patients 81 were white South Africans and seven were patients of mixed race from the West Cape area. Forty eight patients had adult familial hypercholesterolaemia, aged 19-68 years (mean 41 ), of whom 30 (63\%) were women; 16 had juvenile familial hypercholesterolaemia, aged 5-16 years (mean 11), with equal sex distribution; and 24 had mixed hyperlipidaemia, aged 19-69 years (mean 48), of whom $16(67 \%)$ were men. For the three groups the mean cholesterol levels were adult familial hypercholesterolaemia $9 \cdot 72$, juvenile familial hypercholesterolaemia $7 \cdot 81$, and mixed hyperlipidaemia $8.69 \mathrm{mmol} / \mathrm{l}$, and the mean triglyceride levels were adult familial hypercholesterolaemia $1 \cdot 07$, juvenile familial hypercholesterolaemia 0.77 , and mixed hyperlipidaemia $4 \cdot 32 \mathrm{mmol} / \mathrm{l}$.

\section{CONTROLS}

The controls, interviewed and examined in the same way as the patients, were 88 normal volunteers matched for age in decades, sex, and race for each group of patients. They had normal fasting lipid profiles with total chole-

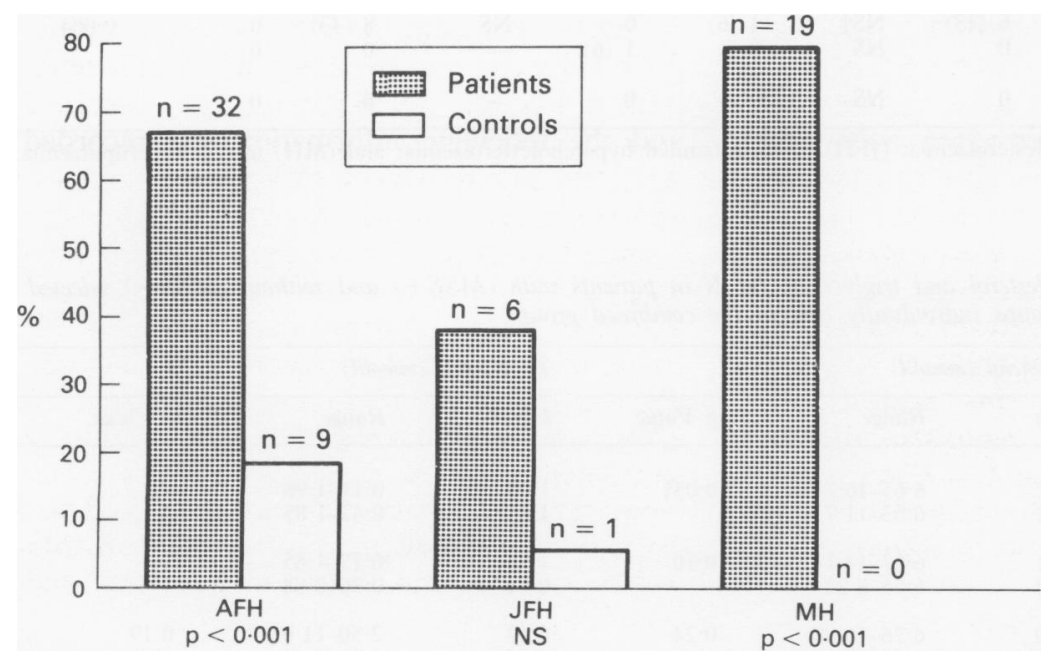

Selected musculoskeletal system manifestations in 48 patients with adult familial hypercholesterolaemia $(A F H), 16$ with juvenile familial hypercholesterolaemia $(\mathcal{F H} H)$, and 24 with mixed hyperlipidaemia $(M H)$ are compared with controls matched for age, sex, and number for each group of patients. The number $(n)$ of patients and controls in each group with manifestations is shown above the bars, and the percentage with manifestations on the y axis. sterol less than the 50 th centile for age ${ }^{12}$ and normal triglyceride levels $(<2 \cdot 0 \mathrm{mmol} / \mathrm{l})$. The normal range for cholesterol and triglyceride levels is the same in the two race groups.

\section{LIPID ANALYSIS}

The plasma was stored at $4^{\circ} \mathrm{C}$ and analysed within 24 hours. The total cholesterol and high density lipoprotein cholesterol levels were measured on a Gilford autoanalyser using the Boehringer Mannheim CHOD-PAP enzymatic method. High density lipoprotein cholesterol was measured after precipitation of the apoprotein B containing lipoproteins with manganese heparin. The non-fasting triglyceride levels were determined by the Boehringer Mannheim enzymatic Peridochrom method. In each instance the Gilford autoanalyser was calibrated against Preciset Cholesterol. Control serum samples used were Precinorm $L$ as an external control and a pooled plasma as an internal control.

\section{STATISTICS}

The $\chi^{2}$ test was used to compare the groups unless less than five manifestations per group were present when Fisher's exact test was used. In determining the difference in cholesterol and triglyceride levels in patients with musculoskeletal system manifestations and those without, logarithmic transformations were applied to remove positive skewness apparent in cholesterol and triglyceride levels.

\section{Results}

ALL MUSCULOSKELETAL SYSTEM MANIFESTATIONS Overall $65(74 \%)$ patients and $50(57 \%)$ controls had one or more musculoskeletal system manifestations $(p=0.026)$. The manifestations included those which previous studies have shown to be associated with hyperlipidaemia and others such as osteoarthritis, rotator cuff syndrome, epicondylitis, and meniscal tears. Three patients aged 10-18 years and none of the controls were hypermobile (a score of 4 or more on Beighton's 9 point scale ${ }^{13}$ ). None had features of the hypermobility syndrome. Gout was present in four patients with mixed hyperlipidaemia and in none of the other patient groups or controls.

\section{MUSCULOSKELETAL SYSTEM MANIFESTATIONS AND HYPERLIPIDAEMIA}

Fifty seven $(65 \%)$ of the 88 patients and 10 (11\%) controls had one or more of the musculoskeletal system manifestations claimed to be associated with hyperlipidaemia $(p<0.001)$, namely tendon xanthomas, tendo Achillis tendinitis, transient Achilles tendon pain, oligoarthritis and migratory polyarthritis. One or more of these manifestations occurred in significantly more patients with adult familial hypercholesterolaemia and mixed hyperlipidaemia than controls (figure). The difference in patients with juvenile familial hypercholesterolaemia and controls was not significant. 
Table 1 gives the prevalence of the individual manifestations. Tendon xanthomas and tendo Achillis tendinitis were significantly higher in patients with adult familial hypercholesterolaemia and mixed hyperlipidaemia and oligoarthritis in patients with mixed hyperlipidaemia. Two patients with juvenile familial hypercholesterolaemia aged 14 and 15 years had palpable tendo Achillis xanthomas, four had had tendo Achillis tendinitis, all after the age of 14 years, and one had a vague history of oligoarthritis.

Transient tendo Achillis pain and migratory polyarthritis were rare in all three groups. Recurrent transient tendo Achillis pain was recorded in one patient with adult familial hypercholesterolaemia and in none with mixed hyperlipidaemia or juvenile familial hypercholesterolaemia. Most episodes occurred on rising from bed, were momentary, and were noninflammatory. Five patients, all with adult familial hypercholesterolaemia, had a history of polyarthritis/polyarthralgia. Only one of these patients described the episodes as migratory. He had had four episodes in three years, each lasting two to 14 days and unaccompanied by inactivity stiffness or inflammatory changes. None of these patients had clinical evidence of rheumatoid arthritis or other arthropathies when examined.

Forty one (47\%) of the 88 patients had xanthomas. Of these $40(98 \%)$ affected one or both Achilles tendons. Other sites were the hand extensors in $12(29 \%)$, the finger flexors in one $(2 \%)$, and the patellar ligament in one $(2 \%)$ patient. All but one of the latter patients also had tendo Achillis. None of the controls had xanthomas.

There was a significant association between tendo Achillis tendinitis and tendo Achillis xanthomas. Twenty eight $(32 \%)$ of the 88 patients had a history of tendo Achillis tendinitis of whom $22(79 \%)$ had palpable tendo Achillis xanthomas and six $(21 \%)$ did not $(p<0.001)$. All four patients with juvenile familial hypercholesterolaemia with tendo Achillis tendinitis attributed the attacks to injuries. In the adults, an association with injury was less obvious. Tendo Achillis tendinitis developed for the first time in the late teens in most patients. The attacks were recurrent in most instances, spanned a period of months or years, and in some were bilateral. In contrast, tendo Achillis tendinitis was unilateral in the three controls and was not recurrent. The attacks were attributed to an injury in two of the controls and to wearing boots in the third.

\section{MUSCULOSKELETAL SYSTEM MANIFESTATIONS} AND LIPID LEVELS

Table 2 gives the pretreatment cholesterol and triglyceride levels in patients with and without musculoskeletal system manifestations and the significance of these. The significance of triglyceride levels in the adult familial hypercholesterolaemia and juvenile familial hypercholesterolaemia groups was not tested because normal levels were used to establish the groups.

\section{ONSET OF MUSCULOSKELETAL SYSTEM}

MANIFESTATIONS

Thirty nine of the $57(68 \%)$ patients with musculoskeletal system manifestations associated with hyperlipidaemia were able to recall whether their symptoms antedated or followed the diagnosis of hyperlipidaemia. In $24(62 \%)$ of these patients the musculoskeletal system manifestations preceded the diagnosis and in 15 (38\%) they had developed after the diagnosis had been made. The difference was not significant $(p=0 \cdot 69)$.

Table 1 Comparison of individual musculoskeletal manifestations in three groups of hyperlipidaemic patients with those of controls matched for age, sex and number. Results given as number (\%)

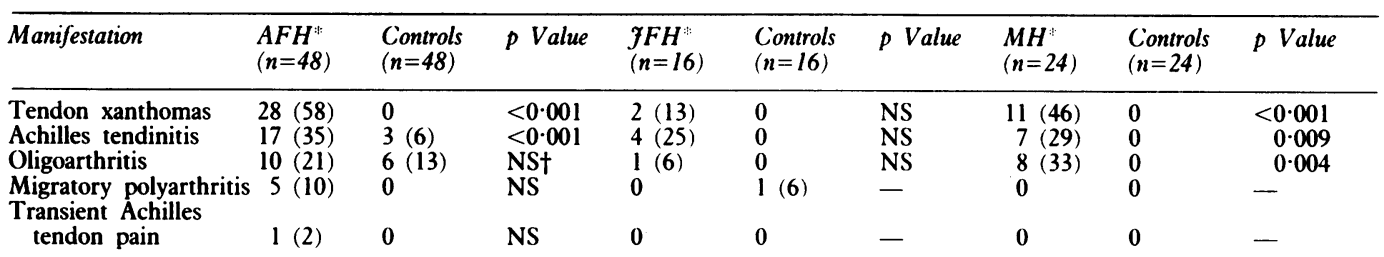

*(AFH) adult familial hypercholesterolaemia; (JFH) juvenile familial hypercholesterolaemia; and (MH) mixed hyperlipidaemia. t(NS) not significant.

Table 2 Comparison of cholesterol and triglyceride levels in patients with (MSS+) and without (MSS-) selected manifestations in the three groups individually and in the combined groups

\begin{tabular}{|c|c|c|c|c|c|c|}
\hline & \multicolumn{3}{|c|}{ Cholesterol (mmol/l) } & \multicolumn{3}{|c|}{ Trigluceride (mmolll) } \\
\hline & Mean & Range & p Value & Mean & Range & p Value \\
\hline $\begin{array}{l}\text { AFH }^{*} \\
\text { MSS }+(n=30) \\
\text { MSS }-(n=18)\end{array}$ & $\begin{array}{r}10 \cdot 21 \\
8 \cdot 92\end{array}$ & $\begin{array}{l}6 \cdot 65-16 \cdot 7 \\
6 \cdot 53-11 \cdot 7\end{array}$ & $0.03 \dagger$ & $\begin{array}{l}1.08 \\
1.06\end{array}$ & $\begin{array}{l}0.11-1.96 \\
0.42-1.85\end{array}$ & - \\
\hline $\begin{array}{l}\mathbf{J F H}^{*} \\
\text { MSS+ }(\mathbf{n}=5) \\
\mathbf{M S S}-(\mathbf{n}=11)\end{array}$ & $\begin{array}{l}9 \cdot 11 \\
7 \cdot 22\end{array}$ & $\begin{array}{l}6 \cdot 57-14 \cdot 14 \\
6 \cdot 55-8 \cdot 22\end{array}$ & $0 \cdot 10$ & $\begin{array}{l}1 \cdot 13 \\
0 \cdot 61\end{array}$ & $\begin{array}{l}0 \cdot 17-1 \cdot 65 \\
0 \cdot 36-0 \cdot 88\end{array}$ & - \\
\hline $\begin{array}{l}\text { MSS+ }(n=17) \\
\text { MSS - }(n=7)\end{array}$ & $\begin{array}{l}9 \cdot 00 \\
7 \cdot 96\end{array}$ & $\begin{array}{l}6 \cdot 76-16 \cdot 90 \\
6 \cdot 54-8 \cdot 94\end{array}$ & $0 \cdot 24$ & $\begin{array}{l}4 \cdot 64 \\
3.53\end{array}$ & $\begin{array}{l}2 \cdot 50-11 \cdot 03 \\
2 \cdot 18-4 \cdot 63\end{array}$ & $0 \cdot 19$ \\
\hline $\begin{array}{l}\text { All three groups } \\
\text { MSS+ }(\mathbf{n}=52) \\
\text { MSS- }(\mathbf{n}=36)\end{array}$ & $\begin{array}{l}9 \cdot 71 \\
8 \cdot 21\end{array}$ & $\begin{array}{l}6 \cdot 57-16 \cdot 90 \\
6 \cdot 53-11 \cdot 70\end{array}$ & $0.001 \dagger$ & $\begin{array}{l}2 \cdot 25 \\
1 \cdot 40\end{array}$ & $\begin{array}{l}0 \cdot 11-11 \cdot 03 \\
0.36-4.63\end{array}$ & 0.07 \\
\hline
\end{tabular}


LIPID LOWERING TREATMENT

Thirty of the 57 patients with musculoskeletal system manifestations associated with hyperlipidaemia were able to state whether their symptoms (mainly tendinitis) had either stopped altogether or had become less frequent and less severe after receiving lipid lowering treatment. Nineteen $(63 \%)$ of these patients felt that they had improved, whereas 11 (37\%) had not noted any difference $(p=0 \cdot 2)$.

Xanthalasma and arcus cornealis were significantly more common in patients with adult familial hypercholesterolaemia than in the controls $(p=0.012$ and $p=0.007$ respectively) and in patients with mixed hyperlipidaemia $(p=0.05)$ for xanthalasma and arcus cornealis.

\section{Discussion}

Epidemiological and other surveys have shown that musculoskeletal system disorders are common in the general population. ${ }^{14}$ The purpose of this controlled study was to investigate the reported associations between musculoskeletal system manifestations and hyperlipidaemia.

Our findings confirm that there is a significant association between xanthomas (particularly of the tendo Achillis) and tendo Achillis tendinitis in patients with adult familial hypercholesterolaemia. In addition, a significant association was shown between xanthomas, tendo Achillis tendinitis, and oligoarthritis in patients with mixed hyperlipidaemia. We found no association, however, between either migratory polyarthritis or transient tendo Achillis pain in any of the groups compared with controls.

There was no significant difference in the number of patients with juvenile familial hypercholesterolaemia with musculoskeletal system manifestations compared with controls. The juvenile familial hypercholesterolaemia group is small, however, and firm conclusions from statistical analysis may be inappropriate. It is probably more pertinent to note that several of the group did have manifestations and that these developed in their late teens, unlike homozygous familial hypercholesterolaemia in which they occurred earlier. This suggests that because of the lower lipid levels in heterozygous familial hypercholesterolaemia a longer period of time is necessary for the development of symptoms.

We showed a significant association between pretreatment cholesterol levels in the patients with adult familial hypercholesterolaemia and in the three groups combined, further suggesting a link between cholesterol levels and the risk of developing musculoskeletal system manifestations. Although not significant, there is some indication that increased triglyceride levels may also predispose to musculoskeletal system manifestations.

Our study confirms the findings in other studies that musculoskeletal system manifestations may antedate the diagnosis of hyperlipidaemia. ${ }^{24}$ Recognition of these manifestations, particularly in children and in subjects with a strong family history of ischaemic heart disease, may contribute to early diagnosis and appropriate treatment of the underlying metabolic disorder.

There are difficulties with the classification of the hyperlipidaemias. Our data suggest that although tendon xanthomas are highly suggestive of familial hyperlipidaemia (Frederickson type IIA), they are often absent in young patients with familial hyperlipidaemia. The diagnosis of familial hyperlipidaemia should therefore not be dependent on xanthomas in young people. In addition, a subject with familial hyperlipidaemia may also have hypertriglyceridaemia due to mixed hyperlipidaemia or to diabetes mellitus, excessive alcohol consumption, obesity, or the use of drugs such as $\beta$ blockers.

The pathogenesis of the musculoskeletal system manifestations in hyperlipidaemia is not fully understood. There is evidence suggesting that the arthropathy is a true inflammatory synovitis, ${ }^{2}$ is periarticular, ${ }^{6}$ or that it is induced by crystals. Cholesterol clefts have been shown in xanthomas ${ }^{15}$ and positively birefringent suspected lipid crystals identified in fluid aspirated from a retrocalcaneal bursa and a tendo Achillis nodule in a patient with hypercholesterolaemia. ${ }^{16}$

It has also been suggested that tendinitis may be precipitated by unaccustomed exertion. ${ }^{3}$ Most of our patients did not associate episodes of tendo Achillis tendinitis with exertion. The significant association between tendo Achillis tendinitis and xanthomas in our study suggests that the xanthomas themselves may play a part in the pathogenesis, possibly by producing an inflammatory reaction on a mechanical basis.

In our study the musculoskeletal system manifestations of some patients improved after lipid lowering treatment. To establish whether control of the hyperlipidaemia will alleviate the musculoskeletal system manifestations requires long term prospective studies relating serial lipid profiles to musculoskeletal system manifestations.

We thank Professor H F H Weich, head of the department of cardiology for permission to study patients attending the lipid clinic and Professor J J F Taljaard and his staff, department of chemical pathology for performing the lipid profiles.

1 Khachadurian A K. Migratory polyarthritis in familia hypercholesterolaemia (Type II hyperlipoproteinemia) Arthritis Rheum 1968; 11: 385-93.

2 Glueck C J, Levy R I, Fredrickson D S. Acute tendinitis and arthritis. A presenting symptom of familial Type II hyperlipoproteinemia. FAMA 1968; 206: 2895-7.

3 Goldman J A, Glueck C J, Abrams N R, Steiner P, Herman H. Musculoskeletal disorders associated with Type IV hyperlipoproteinaemia. Lancet 1972; ii: 449-52.

4 Shapiro J R, Fallat R W, Tsang R C, Glueck C J. Achilles tendinitis and tenosynovitis. A diagnostic manifestation of familial Type II hyperlipoproteinemia in children. $A m, \mathcal{F}$ Dis Child 1974; 128: 486-90.

5 Buckingham R B, Bole G G, Bassett D R. Polyarthritis associated with Type IV hyperlipoproteinemia. Arch Inter Med 1975; 135: 286-90.

6 Rooney P J, Third J, Madkour M M, Spencer D, Dick W C. Transient polyarthritis associated with familial hyperbetalipoproteinaemia. $Q \mathcal{F}$ Med 1978; 187: 249-59

7 Mathon G, Gagné C, Brun D, Lupien P, Moorjani S. Articular manifestations of familial hypercholesterolaemia. Ann Rheum Dis 1985; 44: 599-602.

8 Rimon D, Cohen L. Hypercholesterolemic (Type II hyperlipoproteinemic) arthritis. Case report. 7 Rheumatol i989; 16: 703-5.

9 Welin L, Larsson B, Svärdsudd K, Tibblin G. Serum lipids lipoproteins and musculoskeletal disorders among 50- and 60-year-old men. Scand 7 Rheumatol 1978; 7:7-12. 
10 Struthers G R, Scott D L, Bacon P A, Walton K W. Musculoskeletal disorders in patients with hyperlipidemia. Ann Rheum Dis 1983; 42: 519-23.

11 Wysenbeck A J, Shani E, Beigel Y. Musculoskeletal manifestations in patients with hypercholesterolemia. $\mathcal{F}$ R heumatol 1989; 16: 643-5.

12 Rossouw J E, Steyn K, Berger G M B, et al. Action limits for serum total cholesterol. A statement for the medical profession by an ad hoc committee of the Heart Foundation of Southern Africa. S Afr Med F 1988; 73: 693-700.

13 Beighton P, Solomon L, Soskolne C L. Articular mobility in an African population. Ann Rheum Dis 1973; 32: 413-8.
14 Wood P H N. The basis of rheumatological practice, including nomenclature and classification. In: Scott $\mathrm{J} \mathrm{T}$ ed. Copeman's textbook of the rheumatic diseases. London: Churchill Livingstone, 1986: 19-58.

15 Fahey J J, Stark H H, Donovan W F, Drennan D B. Xanthoma of the Achilles tendon. Seven cases with familial hyperbetalipoproteinemia. J Bone foint Surg [Am] 1973; 55: 1197-221.

16 Schumacher H R, Michaels R. Recurrent tendinitis and Achilles tendon nodule with positively birefringent crystals in a patient with hyperlipoproteinemia. 7 R heumatol 1989 ; in a patient $1387-9$.

2

卫刃 